\title{
Confocal microscopy findings in deep anterior lamellar keratoplasty performed after Descemet's stripping automated endothelial keratoplasty
}

This article was published in the following Dove Press journal:

Clinical Ophthalmology

16 January 2014

Number of times this article has been viewed

\author{
Audrey Pang ${ }^{1,2}$ \\ Karim Mohamed-Noriega' \\ Anita S Chan ${ }^{1,3-5}$ \\ Jodbhir S Mehta ${ }^{1,3}$ \\ 'Singapore National Eye Centre, \\ ${ }^{2}$ Department of Ophthalmology, Tan \\ Tock Seng Hospital, ${ }^{3}$ Singapore Eye \\ Research Institute, ${ }^{4}$ Department of \\ Histopathology, Pathology, Singapore \\ General Hospital, ${ }^{5}$ Department of \\ Ophthalmology, Yong Loo Lin School \\ of Medicine, National University of \\ Singapore, Singapore
}

Background: This study describes the in vivo confocal microscopy findings in two patients who had deep anterior lamellar keratoplasty (DALK) following Descemet's stripping automated endothelial keratoplasty (DSAEK).

Methods: The study reviewed the cases of two patients who first underwent DSAEK followed by DALK when their vision failed to improve due to residual stromal scarring. In the first case, a DSAEK was performed for a patient with pseudophakic bullous keratopathy. After surgery, the patient's vision failed to improve satisfactorily due to residual anterior stromal opacity and irregularity. Subsequently, the patient underwent a DALK. The same two consecutive operations were performed for a second patient with keratoconus whose previous penetrating keratoplasty had failed and had secondary graft ectasia. In vivo confocal microscopy was performed 2 months after the DALK surgery in both cases.

Results: At 3 months after DALK, the best-corrected visual acuity was $6 / 30$ in case 1 and $6 / 24$ in case 2 . In vivo confocal microscopy in both cases revealed the presence of quiescent keratocytes in the stroma layers of the DSAEK and DALK grafts, which was similar in the central and peripheral cornea. There was no activated keratocytes or haze noted in the interface between the grafts.

Conclusion: Our short-term results show that performing a DALK after a DSAEK is an effective way of restoring cornea clarity in patients with residual anterior stromal opacity. In vivo confocal microscopy showed that there were no activated keratocytes seen in the interface of the grafts, which suggests that optimal visual acuity may be obtained with minimal interface haze.

Keywords: cornea, transplant, keratocytes, histology

\section{Introduction}

Over the past 10 years, selective replacement of diseased layers of the cornea in lamellar keratoplasty has been increasingly performed over penetrating keratoplasty (PK). Descemet's stripping automated endothelial keratoplasty (DSAEK) provides more rapid visual improvement in patients with corneal endothelial disease, while deep anterior lamellar keratoplasty (DALK) eliminates the risk of endothelial rejection in patients with corneal stromal disease. ${ }^{1}$

Busin et al first reported a technique of performing a DALK after DSAEK surgery in two patients who had residual anterior stromal opacities. ${ }^{2}$ He showed that a DALK instead of a PK can be performed to preserve the DSAEK graft in patients who have residual cornea opacities. This maintains the structural integrity of the eye and reduces the risk of intraocular complications. In our study, we performed a DALK after DSAEK with a modified technique which preserved the host's Descemet's membrane (DM) in
Correspondence: Audrey Pang Department of Ophthalmology, Tan Tock Seng Hospital, I I Jalan Tan

Tock Seng, 308433 Singapore

Tel +65 9712 6198

Email audreypang@gmail.com 
the periphery of the grafts. It is possible to leave the DM, as we can directly visualize it on the stromal bed of the DSAEK intraoperatively. We also describe the confocal microscopic features seen postoperatively to assess the presence of activated keratocytes and possible interface haze.

\section{Methods}

\section{Case study I}

A 73-year-old female with a history of prior cataract surgery in the left eye more than 30 years previously, and a recent secondary anterior chamber intraocular lens implantation 5 years ago, presented to our clinic in 2011 with left blurring of vision due to pseudophakic bullous keratopathy (Figure 1A). At presentation, her best-corrected visual acuity (BCVA) was counting fingers at $50 \mathrm{~cm}$ distance. Anterior segment optical coherence tomography (OCT) by Visante (Carl Zeiss, Meditec, Jena, Germany) showed mild diffuse anterior stromal opacity which was inferred to be due to the chronic nature of the bullous edema. An uncomplicated left DSAEK was performed in March 2011 (as described below). However, intraoperatively, it was noted following superficial keratectomy that she had an irregular scar on the anterior stromal surface.

Her BCVA improved to $6 / 60(+1.50 /-0.50 \times 180)$ at 3 months post-DSAEK. Repeat anterior segment OCT scans using the Optovue system (Optovue Inc, Fremont, CA, USA) revealed anterior stroma scarring and mild irregular corneal thinning overlying the scar (Figure 1B). In addition, posterior segment OCT revealed mild chronic macula edema. Since there was no change in the irregular thinning over a year, and due to the presence of a guarded visual prognosis from the chronic macula edema, after counseling, we elected to perform a DALK 14 months after the DSAEK surgery.

\section{Case study 2}

A 38-year-old male with bilateral keratoconus, a history of bilateral PKs, and a right trabeculectomy more than 10 years previously, had been on regular follow-up in our clinic.

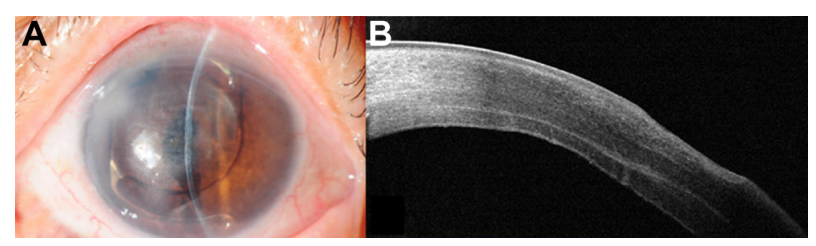

Figure I Left pseudophakic bullous keratopathy of case I at presentation (A) and anterior segment OCT after DSAEK showing mild anterior stromal opacity and irregularity (B).

Abbreviations: DSAEK, Descemet's stripping automated endothelial keratoplasty; OCT, optical coherence tomography.
In September 2012, his BCVA was 6/12 $(+2.75 /-4.00 \times 5)$ in the right eye and $6 / 9($ plano/ $-5.00 \times 40)$ in the left eye. However, he developed left corneal graft failure and was noted to have inferior graft ectasia in January 2013 (Figure 2). His BCVA dropped to counting fingers at 1 meter distance. He also had a left cataract. He underwent a DSAEK and phacoemulsification surgery in January 2013. During the DSAEK procedure, following the air tamponade, his inferior graft ectasia became worse. In view of this, he underwent a left DALK 1 week later.

\section{Surgical procedures}

The DSAEK was performed under general anesthesia using a previously described technique. ${ }^{3}$ The DM was stripped under air through a temporal sclera incision in case 1 and no stripping was performed in case 2. An inferior peripheral iridectomy was performed. Four venting incisions were made. The lamellar graft was harvested from the donor cornea using the Moria ALTK (automated lamellar therapeutic keratoplasty) microkeratome (Moria Inc., Doylestown, PA, USA).

The diameter of the lamellar graft was $8.75 \mathrm{~mm}$ in case 1 and $9.0 \mathrm{~mm}$ in case 2. The lamellar graft was inserted into the anterior chamber using the Endoglide DSAEK insertion device (Network Medical Products, Ripon, UK). ${ }^{3}$ Once the graft was inserted, an air bubble was injected into the anterior chamber, and a full air-fill was maintained for 10 minutes. Aqueous fluid was expressed through the venting incisions to ensure good graft-host apposition.

The DALK was performed under general anesthesia. The corneal surface was first marked with a Solan trephine (Medtronics, Inc., Jacksonville, FL, USA). The diameter of the trephine was $7.5 \mathrm{~mm}$ in case 1 and $8.0 \mathrm{~mm}$ in case 2. The size of the graft was chosen to allow at least $0.5 \mathrm{~mm}$ rim of the DSAEK around the DALK. A free-hand manual dissection was

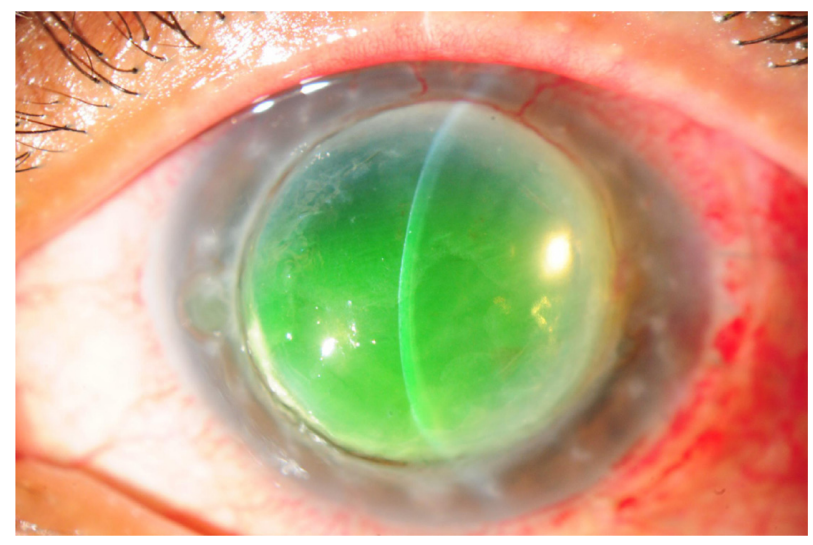

Figure 2 Left penetrating keratoplasty failure due to graft ectasia and recurrent keratoconus in case 2 . 
performed with gentle traction of the recipient anterior stroma until a clear interface could be reached. Once an interface was identified, it was carefully exposed using a marginal lamellar dissector (ASICO, Westmont, IL, USA), and the recipient stroma was cut along the previous trephine mark. Following complete exposure of the interface, we noted that, in fact, the recipient's DM had been split into anterior and posterior layers. In case 1 the central cornea was devoid of any DM following Descemet's stripping as part of the DSAEK procedure. The paracentral/peripheral interface had the presence of the recipient's posterior nonbanded DM. This was visualized intraoperatively as a bright, reflective surface on the interface bed and was confirmed histologically (Figure 3). The anterior banded layer of DM could be visualized on the removed corneal button. In the histological specimen from case 2, the complete posterior nonbanded part of the DM could be seen attached to the DSAEK, and the peripheral interface had remnants of the anterior DM layer, the central anterior layer attached to the recipient's removed cornea. Intraoperatively, the split between the banded and nonbanded part of the DM is only possible since there is DM present on both the recipient tissue and the DSAEK graft following the removal of the corneal button, hence there must be a split in the DM to achieve this. The donor cornea was trephined with a $7.5 \mathrm{~mm}$ and $8.0 \mathrm{~mm}$ trephine (Moria Inc.) in case 1 and case 2 , respectively. The DM was removed before suturing the graft in place with two 10/0 nylon sutures.

\section{Confocal microscopy}

In vivo confocal microscopy was performed at 2 months postoperative using the Heidelberg Retina

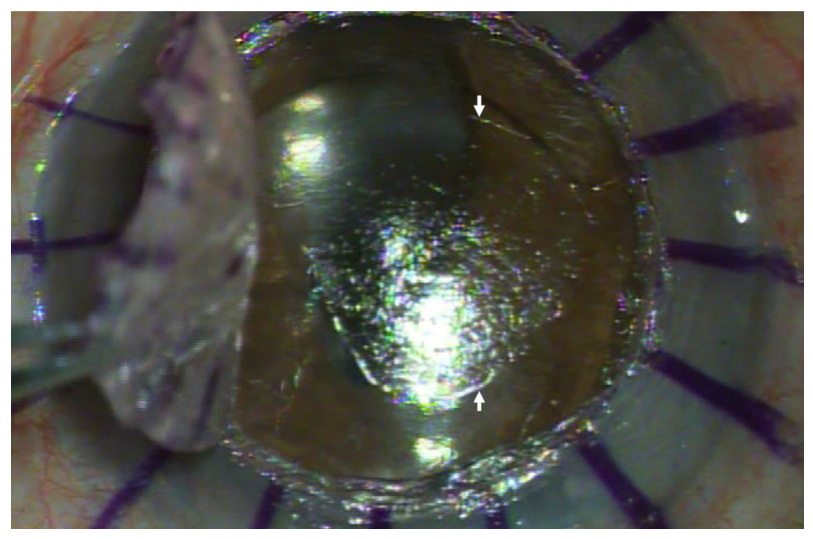

Figure 3 Intraoperative view of Descemet's membrane after anterior host stroma layer is removed during DALK in case I.

Notes: The arrows indicate visible margin of the central area, which was devoid of any DM following Descemet's stripping as part of the previous DSAEK procedure. The peripheral interface beyond the arrows had the presence of the recipient's posterior nonbanded DM.

Abbreviations: DALK, deep anterior lamellar keratoplasty; DM, Descemet's membrane; DSAEK, Descemet's stripping automated endothelial keratoplasty.
Tomography 2 Rostock Cornea Module (HRT 2 RCM; Heidelberg Engineering, Germany). After a drop of contact gel (Vidisic Gel; Bausch and Lomb, Rochester, NY, USA) was applied onto the front of the microscope lens, a TomoCap (Heidelberg Engineering) was mounted onto the holder to cover the microscope lens. The HRT 2 RCM uses a 60× immersion objective lens (Olympus Europa GmbH, Hamburg, Germany) and a $670 \mathrm{~nm}$ diode laser as the light source. The central cornea was examined in layers using the volumetric scan, advancing from the epithelium to the endothelium. Both the central cornea and peripheral cornea between the two grafts were examined. Four examinations of the eye were performed.

\section{Results}

At 3 months postoperative, the case 1 patient's BCVA improved to $6 / 30(-6.00 /-1.00 \times 110)$. Slit lamp biomicroscopy revealed clear corneal grafts in the left eye (Figure 4A). Posterior segment OCT revealed the presence of chronic mild cystoid macula edema. Anterior segment OCT showed a regular corneal surface and a smooth interface between the DSAEK and DALK grafts (Figure 4B).
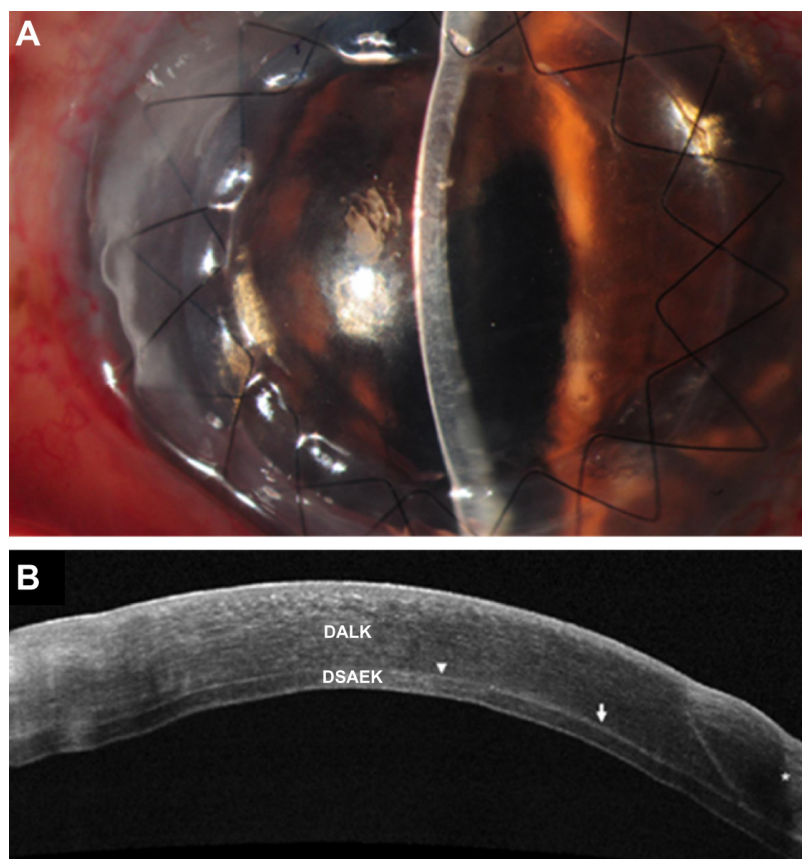

Figure 4 Postoperative image of the left eye in case I after both DSAEK and $\operatorname{DALK}(\mathbf{A})$ and anterior segment OCT showing a smooth interface between the DALK and DSAEK grafts respectively (B).

Notes: The arrowhead points to the central cornea area that was devoid of DM, and the arrow indicates the paracentral area where the host DM was still present between the two lamellar grafts (B).

Abbreviations: DALK, deep anterior lamellar keratoplasty; DM, Descemet's membrane; DSAEK, Descemet's stripping automated endothelial keratoplasty; OCT, optical coherence tomography. 
The host DM was clearly seen at the periphery, between the two lamellar grafts.

In case 2, the patient's BCVA improved to $6 / 24$ $(+4.50 /-7.00 \times 60)$ at 3 months postoperative. His suboptimal visual acuity was due to coexisting advanced glaucoma. Slit lamp biomicroscopy revealed clear cornea grafts in the left eye (Figure 5A). Anterior segment OCT also showed a regular corneal surface and a smooth interface between the DSAEK and DALK grafts (Figure 5B).

In both cases, the histology of the host corneal button showed remnants of the anterior DM of the host cornea (Figure 6). At the periphery, the host DM had split, with the anterior portion attached to the host, leaving the posterior portion adhered to the DSAEK graft.

In vivo confocal microscopy of the central cornea in both patients demonstrated normal quiescent keratocytes in the anterior and posterior stromal layers of the DSAEK and DALK, respectively (Figures 7 and 8).

Typically, quiescent keratocytes were seen as regular oval-shaped cells with a bright nucleus and were well separated from each other. No activated keratocytes were seen in the stroma or the interface between the grafts. No inflammatory cells or corneal nerves were seen in the DALK and DSAEK grafts.

The interface between the grafts was identified as an amorphous, acellular zone with variable background
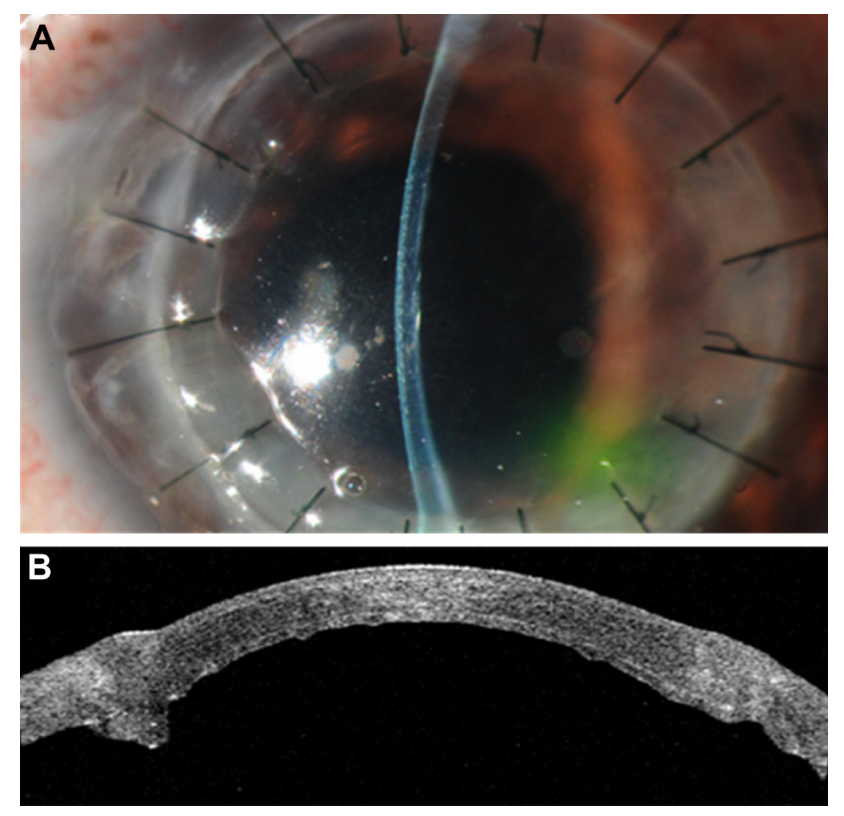

Figure 5 Postoperative image of the left eye in case 2 after both DSAEK and DALK (A) and anterior segment OCT showing a smooth interface between the DALK and DSAEK grafts respectively (B).

Abbreviations: DALK, deep anterior lamellar keratoplasty; DSAEK, Descemet's stripping automated endothelial keratoplasty; OCT, optical coherence tomography.

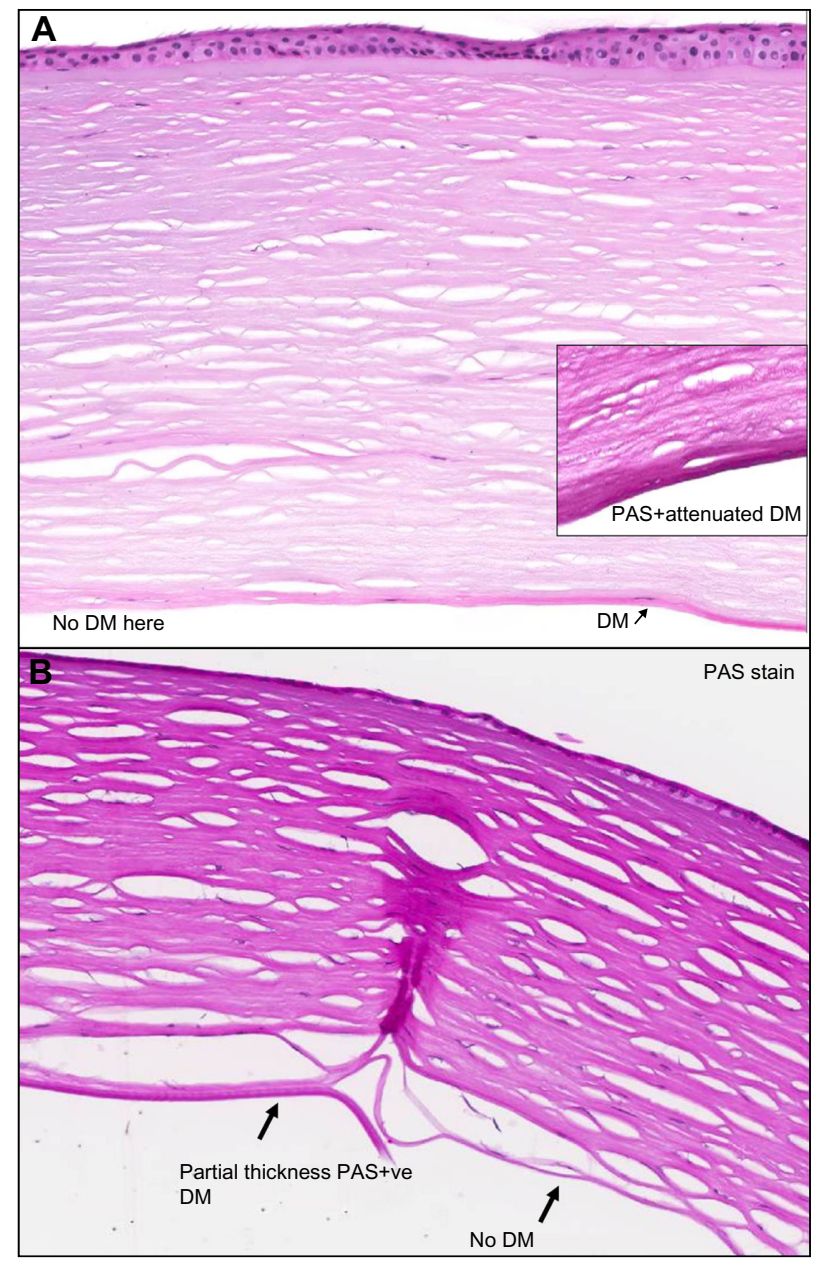

Figure 6 Histology of the host corneal button from the DALK surgery in both case I (A) and case 2 (B) shows that the host Descemet's membrane had split at the periphery, with the anterior portion attached to the host, leaving the posterior portion adhered to the DSAEK graft.

Abbreviations: DALK, deep anterior lamellar keratoplasty; DM, Descemet's membrane; DSAEK, Descemet's stripping automated endothelial keratoplasty; PAS, periodic acid-Schiff.

extracellular reflectivity. A few hyperreflective birefringent particles, which were likely debris, were observed at the interface. Microfolds within the graft stroma near the interface were seen as dark hyporeflective striae.

At the periphery of the two corneal grafts there was a similar finding of quiescent keratocytes noted in the stroma of the DALK and DSAEK grafts (Figures 9 and 10). There was neither activated keratocytes nor haze noted at the interface. Small hyperreflective particles corresponding to debris were also noted in the interface.

\section{Discussion}

It is occasionally difficult to predict the visual significance of stroma opacities in edematous corneas, which affects the choice between lamellar and PK procedures. As previously 


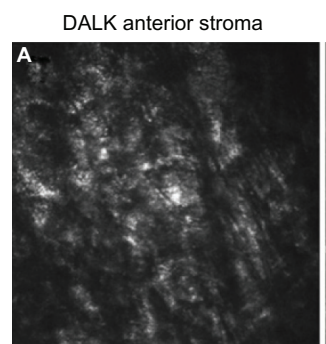

Interface

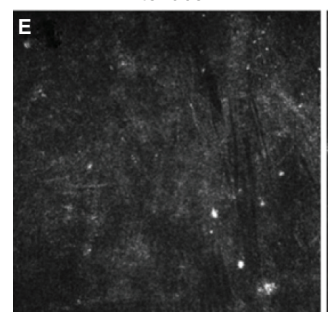

DALK posterior stroma

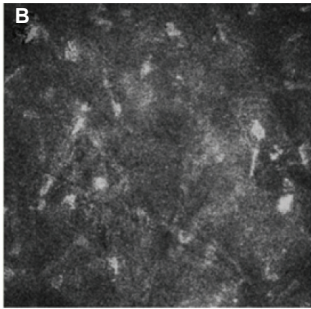

Interface DSAEK

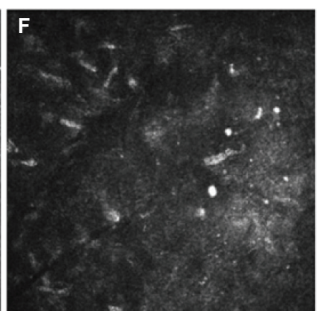

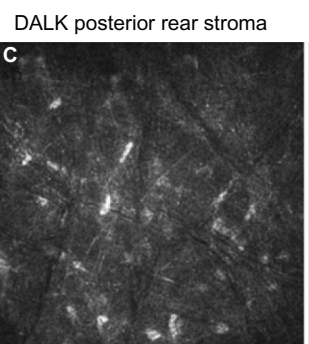

DSAEK stroma

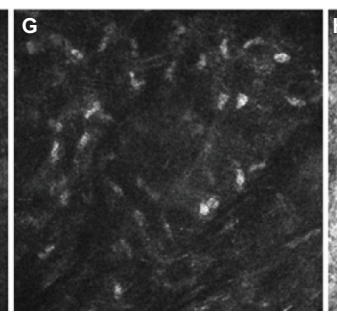

Interface DALK

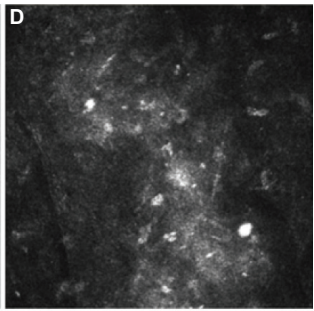

EM/fold/DM/DSAEK stroma

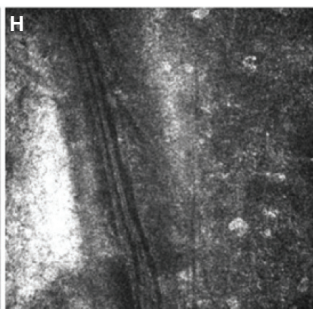

Figure 7 In case I, in vivo confocal microscopy of the central corneal interface between the DALK and DSAEK grafts showed quiescent keratocytes.

Note: No activated keratocytes or interface haze was noted. Confocal images (slides A-H) of progressive central corneal layers from the anterior stroma to the endothelium showing quiescent keratocytes in all stromal layers and typical folds of lamellar grafts.

Abbreviations: DALK, deep anterior lamellar keratoplasty; DM, Descemet's membrane; DSAEK, Descemet's stripping automated endothelial keratoplasty; EM, endothelium.

described by Busin et al, the approach of performing a DALK after DSAEK surgery in patients is an effective treatment in patients who fail to achieve satisfactory visual improvement after DSAEK. ${ }^{2}$ Previously Busin et al used a Hessburg-Baron trephine to create a partial-thickness trephination aligned with the edge of the DSAEK graft. The incision was deepened manually until the stromal surface of the DSAEK graft was reached. No adhesion was noted between the host and the DSAEK graft, and the donor tissue was punched to size and sutured in place.

In our study, we sized our partial-thickness trephine to $1 \mathrm{~mm}$ smaller than the DSAEK graft. A free-hand manual dissection was performed until a clear interface was identified and carefully exposed using a marginal lamellar dissector. After complete exposure of the interface, we noted that the recipient's DM in the periphery had been split as described in the surgical procedures above. The central cornea was devoid of any DM as expected following Descemet's stripping during the DSAEK procedure. The peripheral interface had the presence of the recipient's remnant posterior DM, which was visualized intraoperatively as a bright, reflective surface on the interface bed. Our cases suggest that there is stronger adhesion between the DSAEK graft stromal surface and the posterior nonbanded DM than between the individual DM layers.

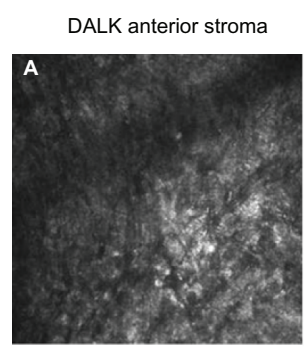

Interface

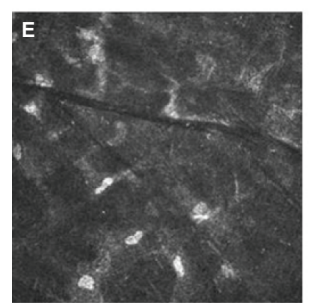

DALK posterior stroma

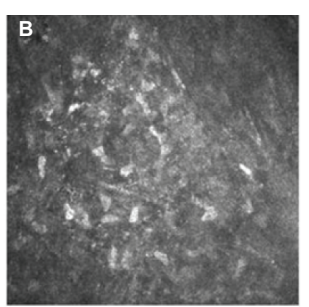

Interface DSAEK

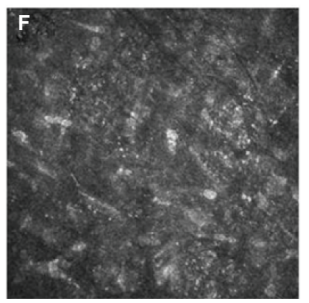

DALK posterior rear stroma

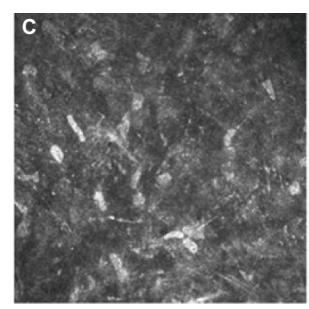

DSAEK stroma

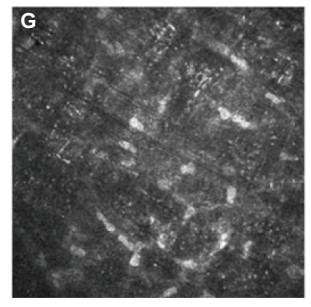

Interface DALK

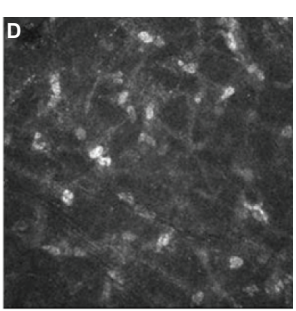

EM/fold/DM/DSAEK stroma

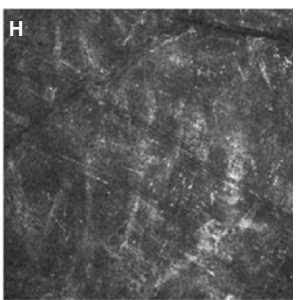

Figure 8 In case 2, in vivo confocal microscopy of the central corneal interface between the DALK and DSAEK grafts showed quiescent keratocytes.

Note: No activated keratocytes or interface haze was noted. Confocal images (slides A-H) of progressive central corneal layers from the anterior stroma to the endothelium showing quiescent keratocytes in all stromal layers and typical folds of lamellar grafts.

Abbreviations: DALK, deep anterior lamellar keratoplasty; DM, Descemet's membrane; DSAEK, Descemet's stripping automated endothelial keratoplasty; EM, endothelium. 


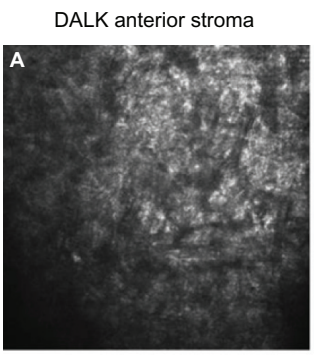

Interface DM

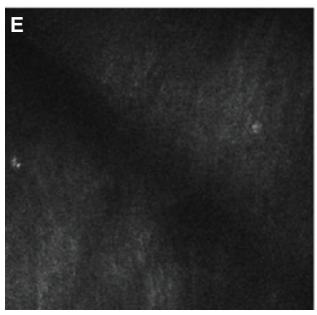

DALK posterior stroma

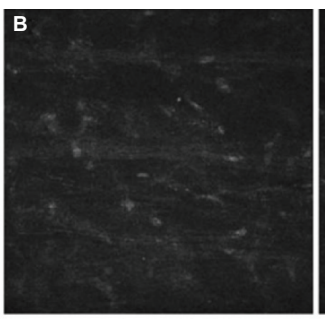

Interface DM/DSAEK

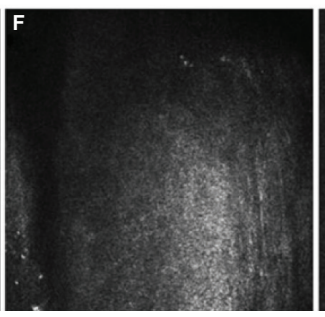

DALK posterior rear stroma

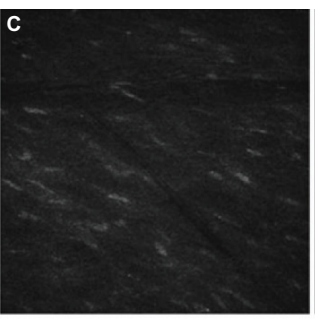

Interface DSAEK

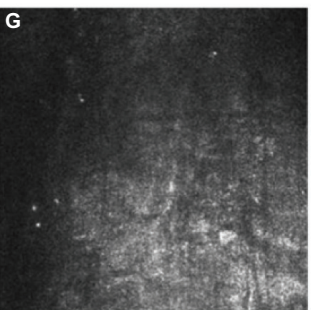

Interface DALK

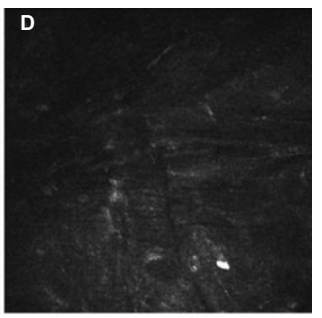

DSAEK stroma

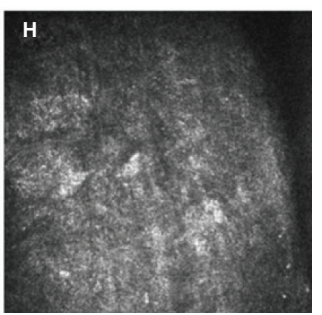

Figure 9 In case I, in vivo confocal microscopy of the peripheral corneal interface between the DALK and DSAEK grafts was similar to the central cornea interface. Note: No activated keratocytes were noted. Confocal images (slides A-H) of progressive corneal layers of the peripheral grafts from the anterior DALK stroma to the posterior DSAEK stroma showing quiescent keratocytes in all layers and interface of lamellar grafts.

Abbreviations: DALK, deep anterior lamellar keratoplasty; DM, Descemet's membrane; DSAEK, Descemet's stripping automated endothelial keratoplasty.

Normal corneal stroma consists of a dense network of collagen fibers, keratocytes, and proteoglycans. Keratocytes in the stroma are usually mitotically quiescent and have numerous intercellular contacts. During corneal wound healing, keratocyte apoptosis occurs in the region of injury, and repopulation often occurs by cells that become activated to undergo mitosis and migrate to repopulate the apoptotic area. ${ }^{4}$ Activated keratocytes involved in cellular repair have been previously identified in corneal transplants by their large, brightly reflective appearance associated with visible cytoplasmic processes. ${ }^{5}$ Confocal microscopy has shown the presence of active keratocytes in recipient stroma after DSEK. ${ }^{6}$ These prior studies show that activated keratocytes play a role in the repair of the extracellular matrix; however, the altered composition of the extracellular matrix and haphazard deposition of keratocytes scatter light and is believed to contribute to postoperative haze formation. ${ }^{7}$

Ultrastructural analysis of the grafts in our cases was performed using in vivo confocal microscopy. Our study showed that there were no activated keratocytes noted in the stroma of both the DALK and DSAEK grafts, especially the interfaces between the grafts. This microscopic finding was

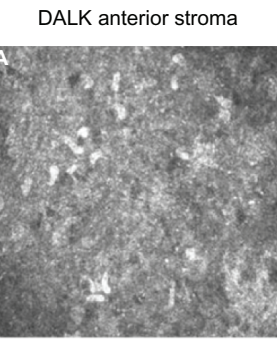

Interface DM

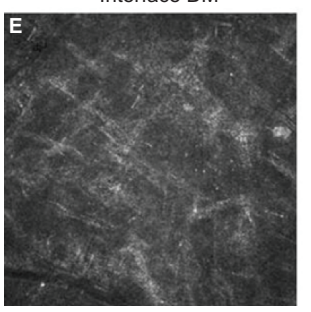

DALK posterior stroma

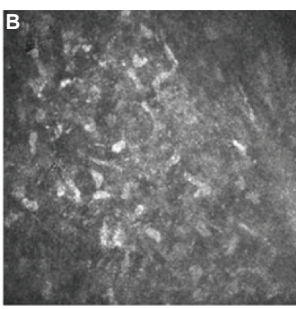

Interface DM/DSAEK

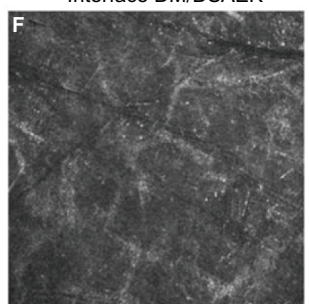

DALK posterior rear stroma

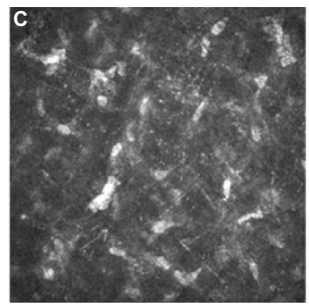

Interface DSAEK

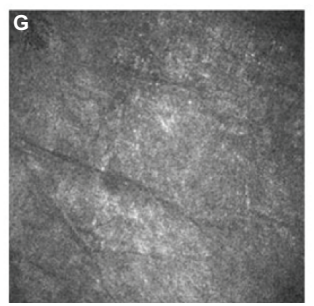

Interface DALK

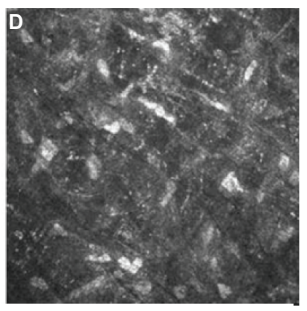

DSAEK stroma

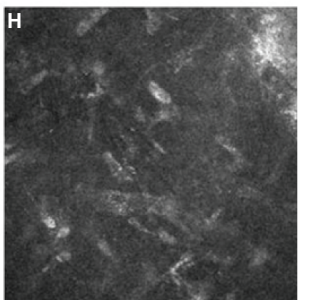

Figure 10 In case 2, in vivo confocal microscopy of the peripheral corneal interface between the DALK and DSAEK grafts was similar to the central cornea interface. Note: No activated keratocytes were noted. Confocal images (slides A-H) of progressive corneal layers of the peripheral grafts from the anterior DALK stroma to the posterior DSAEK stroma showing quiescent keratocytes in all layers and interface of the lamellar grafts.

Abbreviations: DALK, deep anterior lamellar keratoplasty; DM, Descemet's membrane; DSAEK, Descemet's stripping automated endothelial keratoplasty. 
similar both in the center and periphery of the grafts. Similar findings of quiescent keratocytes have been described after individual manual deep lamellar keratoplasty, ${ }^{5}$ DSAEK,${ }^{8}$ DALK, ${ }^{9}$ and laser-assisted in situ keratomileusis ${ }^{10}$ surgeries. In the periphery of the grafts, we did not expect the presence of postoperative corneal haze, as this is analogous to the interface in a DM baring DALK. Confocal microscopy in our study has also shown that there were no activated keratocytes in the central area as well as the periphery despite having two interfaces in the periphery of case 1 and in both the center and periphery of case 2 . The two interfaces are the donor DALK stroma/host DM interface, and the host DM/ DSAEK stromal interface. We recognize that the indications for serial DSAEK and DALK are relatively rare, and this study describes only two cases. More cases will need to be described to show the validity of this procedure. Nevertheless, the absence of activated keratocytes in these two cases provides encouraging evidence, although limited, that the risk of developing corneal haze in patients who have undergone a DALK after DSAEK surgery is low.

\section{Conclusion}

Our results suggest that a DALK performed after DSAEK surgery can aid in the visual rehabilitation of patients with residual stromal scarring. There was minimal evidence of interface haze or scarring.

\section{Disclosure}

The authors report no conflicts of interest in this work.

\section{References}

1. Tan DT, Dart JK, Holland EJ, Kinoshita S. Cornea transplantation. Lancet. 2012;379(9827):1749-1761.

2. Busin M, Beltz J. Deep anterior lamellar keratoplasty after descemet stripping automated endothelial keratoplasty. Cornea. 2011;30(9): $1048-1050$.

3. Khor WB, Mehta JS, Tan DT. Descemet stripping automated endothelial keratoplasty with a graft insertion device: surgical technique and early clinical results. Am J Ophthalmol. 2011;151(2):223-232. e2.

4. Mohan RR, Hutcheon AE, Choi R, et al. Apoptosis, necrosis, proliferation, and myofibroblast generation in the stroma following LASIK and PRK. Exp Eye Res. 2003;76(1):71-87.

5. Bourne WM. Cellular changes in transplanted human corneas. Cornea. 2001;20(6):560-569.

6. Savastano A, Sbordone S, Piccirillo V, et al. Confocal microscopy after descemet stripping endothelial keratoplasty: a case report. Cornea. 2009;28(5):570-574.

7. Zieske JD. Extracellular matrix and wound healing. Curr Opin Ophthalmol. 2001;12(4):237-241.

8. Kobayashi A, Mawatari Y, Yokogawa H, Sugiyama K. In vivo laser confocal microscopy after descemet stripping with automated endothelial keratoplasty. Am J Ophthalmol. 2008;145(6):977-985.

9. Feizi S, Javadi MA, Kanavi MR. Cellular changes of donor corneal tissue after deep anterior lamellar keratoplasty versus penetrating keratoplasty in eyes with keratoconus: a confocal study. Cornea. 2010;29(8):866-870.

10. Perez-Gomez I, Efron N. Confocal microscopic evaluation of particles at the corneal flap interface after myopic laser in situ keratomileusis. J Cataract Refract Surg. 2003;29(7):1373-1377.
Clinical Ophthalmology

\section{Publish your work in this journal}

Clinical Ophthalmology is an international, peer-reviewed journal covering all subspecialties within ophthalmology. Key topics include: Optometry; Visual science; Pharmacology and drug therapy in eye diseases; Basic Sciences; Primary and Secondary eye care; Patien Safety and Quality of Care Improvements. This journal is indexed on

Submit your manuscript here: http://www.dovepress.com/clinical-ophthalmology-journal

\section{Dovepress}

PubMed Central and CAS, and is the official journal of The Society of Clinical Ophthalmology (SCO). The manuscript management system is completely online and includes a very quick and fair peer-review system, which is all easy to use. Visit http://www.dovepress.com/ testimonials.php to read real quotes from published authors. 